\title{
Relación de la edad con el manejo de los pacientes diagnosticados de neoplasia en un Servicio de Medicina Interna
}

\author{
E. RUIZ, J. ALEGRE, O. LEN, L. Ma ARMADANS *, J. RECIO, C. ALEMÁN, \\ T. FERNÁNDEZ DE SEVILLA \\ Servicios de Medicina Interna y Medicina Preventiva y *Servicio de Epidemiología. \\ Hospital General Universatario Vall d'Hebron. Barcelona
}

\author{
REPERCUSSION OF AGE ON MANAGEMENT OF NEOPLASTIC \\ PATIENTS IN AN INTERNAL MEDICINE SERVICE
}

\section{RESUMEN}

Objetivo: Valorar la relación de la edad con el manejo de los pacientes diagnosticados de neoplasia en un servicio de Medicina Interna.

Pacientes y métodos: Estudio prospectivo de 388 pacientes diagnosticados de cáncer en un Servicio de Medicina Interna. Se evalúan las características clínicas, procedimientos diagnósticos, tipos de neoplasia y estadiaje, derivación al alta y tratamiento. Se realiza un estudio comparativo basado en la edad, pacientes de 65 años o mayores frente a los más jóvenes.

Resultados: Los 388 pacientes diagnosticados de cáncer supusieron un $12 \%$ de los ingresos en nuestro servicio. El $62 \%$ tenían $\geq 65$ años. El síndrome constitucional fue la sintomatología más frecuente asociada y el principal motivo de consulta, siendo más relevante en los $\geq 65$ años. El cáncer de pulmón fue el más frecuente en los hombres y las neoplasias hematológicas en las mujeres. Los enfermos $\geq 65$ años fueron controlados con más frecuencia por Medicina Interna, médico de cabecera y Servicio de Asistencia Paliativa a Domicilio $(\mathrm{P}<0,05)$ y recibieron tratamiento únicamente paliativo en un mayor porcentaje de casos (50\% vs $37 \%, \mathrm{p}=0,001)$.

Conclusiones: La incidencia de neoplasias en nuestro servicio fue alta $(12 \%)$, con un alto porcentaje de pacientes en fases avanzadas de la enfermedad (83\%). La edad no se relacionó con el manejo diagnóstico ni demostró ser determinante en el estadiaje, pero sí en el tratamiento, puesto que los pacientes $\geq 65$ años recibieron con mayor frecuencia tratamiento paliativo.

PALABRAS CLAVE: Neoplasia. Cáncer. Edad.

\begin{abstract}
Aim: To determine whether age is a factor affecting the management of patiens diagnosed with neoplastic disease in an internal medicine ser vice.

Patients and methods: Prospective study of 388 patients diagnosed with cancer in the internal medicine service of a large public health tea ching hospital. We evaluated clinical characteristics, diagnostic proce dures, types and stage of neoplasm, referral after hospital discharge and treatment. A comparative study based on age was performed between patients 65 years older and patients under this age.

Results: The 388 cancer patiens accounted for 12\% of hospital admissions in our service. Among the total, $62 \%$ were $\geq 65$ years old. Constitutional syndrome, the most frequently associated symptom and the main reason for the consultation, was more common in the $\geq 65$ year-old group. Lung cancer was predominant in men and hematologic neoplasms in women. Patients $\geq 65$ were referred more frequently to internal medicine physicians, general practitioners and home palliative assistance services $(p<0.05)$ and they received only palliative treatment in a greater percentage of cases $(50 \% v s .37 \%, p=0.001)$.

Conclusions: The incidence of neoplasms in our service was high $(12 \%)$, with a considerable percentage of patients in advanced phases of the disease (83\%). Age was not related to diagnostic methods nor was it determinant in the staging, but it did have repercussions on treatment; patients $\geq 65$ received palliative treatment alone more often than younger patients.
\end{abstract}

KEY WORDS: Neoplasm. Cancer. Age.

Ruiz E, Alegre J, Len O, Armadans LMa, Recio J, Alemán C, Fernández de Sevilla T. Relación de la edad con el manejo de los pacientes diag nosticados de neoplasia en un Servicio de Medicina Interna. An Med Interna (Madrid) 2002; 19: 506-510.

\section{INTRODUCCIÓN}

El cáncer representa uno de los mayores problemas de salud pública, siendo una causa mayor de morbilidad y mortalidad en todo el mundo $(1,2)$. En la actualidad se estima que aproximadamente $1 / 3$ de la población de los países desarrollados padecerá algún tipo de neoplasia (3). El factor de riesgo más importante para el cáncer es la edad, de manera que más de la mitad de todos los cánceres ocurren en pacientes de 65 años o mayores y el $70 \%$ de las muertes debidas a cáncer tiene lugar en este grupo de edad $(4,5)$. A pesar de ello existe una inesperada infrarrepresentación de los pacientes de 65 años o más en los ensayos sobre el tratamiento del cáncer $(6,7)$. Este hecho conlleva una falta de información real en este grupo de enfermos y por consiguiente un manejo inadecuado (8). De todo ello deriva la

Trabajo aceptado: 10 de junio de 2002

Correspondencia: Eva Ruiz Ruiz. C/ Salvà n 48, $1^{\text {o }}$ - 2 $2^{\mathrm{a}} .08004$ Barcelona 
necesidad de estudios más específicos sobre cancer en gente mayor $(9,10)$.

La mayoría de pacientes oncológicos son valorados inicialmente en servicios de Medicina Interna. A pesar de ello existen escasas referencias sobre su manejo en la práctica clínica diaria y sobre el impacto de la edad en este grupo de pacientes. Por ello, nos propusimos un estudio clínico de los enfermos diagnosticados de cáncer en un Servicio de Medicina Interna. Nuestro objetivo fue valorar la asociación de la edad en este grupo de pacientes, estudiando las características clínicas, métodos de diagnóstico, tipo de neoplasia, estadiaje, derivación al alta y tratamiento inicial.

\section{PACIENTES Y MÉTODOS}

Hemos realizado un estudio prospectivo de 388 pacientes diagnosticados de cáncer en un Servicio de Medicina Interna durante un período de 3 años (enero'97-diciembre'99). Incluimos todos los pacientes diagnosticados de patología tumoral maligna mediante citología, biopsia o ambas durante su estancia en nues- tro servicio. Excluímos aquellos que ya se hallaban afectos de una neoplasia no considerados libres de enfermedad y aquellos en los que no se llegó a un diagnóstico histológico. A todos se les aplicó un protocolo clínico donde se recogieron datos sobre la edad, el sexo, el motivo de ingreso, los síntomas y signos asociados, exploraciones complementarias realizadas, tipo de neoplasia y estadiaje, derivación al alta y tratamiento inicial recibido. Realizamos un análisis comparativo basado en la edad, pacientes $\geq 65$ años respecto los menores de 65 años. Hemos tomado este punto de corte teniendo en cuenta estudios previos (1-4).

Análisis estadístico: Para las variables categóricas se ha realizado el test de $\chi^{2}$ y para las variables cuantitativas el test de $t$ de Student. Se ha considerado significativa una $\mathrm{p}<0,05$.

\section{RESULTADOS}

Los 388 pacientes diagnosticados de neoplasia supusieron un $12 \%$ del total de los ingresos en nuestro servicio de Medicina Interna (3.233 ingresos durante un periodo de 3 años). En la tabla I se recogen las características generales y en función

\section{TABLA I}

\begin{tabular}{|c|c|c|c|c|c|c|c|}
\hline \multicolumn{8}{|c|}{ TABLA I } \\
\hline \multicolumn{8}{|c|}{ CARACTERÍSTICAS DE LO S PACIENTES DIAGNO STICADOS DE CÁNCER } \\
\hline & \multicolumn{2}{|c|}{$\begin{array}{c}\text { Total } \\
n=388\end{array}$} & \multicolumn{2}{|c|}{$\begin{array}{l}\text { Edad }< \\
65 \text { años } \\
n=145\end{array}$} & \multicolumn{2}{|c|}{$\begin{array}{l}\text { Edad } \geq \\
65 \text { años } \\
n=243\end{array}$} & $\mathrm{p}$ \\
\hline $\begin{array}{l}\text { Días ingreso (media } \pm \text { DS) } \\
\text { Hombres } \\
\text { Mujeres }\end{array}$ & $\begin{array}{l}10, \\
283 \\
105\end{array}$ & $\begin{array}{r}+7,8 \\
73 \% \\
27 \%\end{array}$ & $\begin{array}{r}110 \\
35\end{array}$ & $\begin{array}{l}+5,1 \\
76 \% \\
24 \%\end{array}$ & $\begin{array}{r}173 \\
70\end{array}$ & $\begin{array}{l} \pm 9 \\
71 \% \\
29 \%\end{array}$ & $\begin{array}{l}0,02 \\
\text { NS } \\
\text { NS }\end{array}$ \\
\hline $\begin{array}{l}\text { M otivo de ingreso } \\
\text { Síndrome constitucional } \\
\text { Dolor óseo } \\
\text { Dolor abdominal } \\
\text { Dísnea } \\
\text { Derrame pleural } \\
\text { Ascitis } \\
\text { Ictericia } \\
\text { Anemia } \\
\text { Radiografía patológica } \\
\text { Adenopatías } \\
\text { Focalidad Neurológica } \\
\text { Fiebre } \\
\text { O tros }\end{array}$ & $\begin{array}{r}79 \\
55 \\
39 \\
24 \\
9 \\
13 \\
10 \\
12 \\
20 \\
18 \\
14 \\
14 \\
81\end{array}$ & $\begin{array}{r}20 \% \\
14 \% \\
10 \% \\
6 \% \\
2 \% \\
3 \% \\
3 \% \\
3 \% \\
5 \% \\
5 \% \\
4 \% \\
4 \% \\
21 \%\end{array}$ & $\begin{array}{r}17 \\
23 \\
16 \\
7 \\
\\
5 \\
2 \\
4 \\
14 \\
3 \\
9 \\
8 \\
27\end{array}$ & $\begin{array}{r}12 \% \\
16 \% \\
11 \% \\
5 \% \\
3 \% \\
1 \% \\
3 \% \\
10 \% \\
9 \% \\
6 \% \\
5 \% \\
19 \%\end{array}$ & $\begin{array}{r}62 \\
32 \\
23 \\
17 \\
9 \\
9 \\
8 \\
8 \\
6 \\
5 \\
5 \\
6 \\
53\end{array}$ & $\begin{array}{r}26 \% \\
13 \% \\
10 \% \\
7 \% \\
4 \% \\
4 \% \\
3 \% \\
3 \% \\
2 \% \\
2 \% \\
2 \% \\
2 \% \\
22 \%\end{array}$ & $\begin{array}{l}0,001 \\
N S \\
N S \\
N S \\
N S \\
N S \\
N S \\
N S \\
N S \\
N S \\
N S \\
N S \\
N S\end{array}$ \\
\hline $\begin{array}{l}\text { Síntomas y signos } \\
\text { Síndrome constitucional } \\
\text { Dolor óseo } \\
\text { Alt. ritmo deposicional } \\
\text { Dolor abdominal } \\
\text { Vómitos } \\
\text { Síndrome anémico } \\
\text { Hemoptisis } \\
\text { Fiebre } \\
\text { M asa abdominal } \\
\text { Hepatomegalia } \\
\text { Adenopatías } \\
\text { Focalidad neurológica }\end{array}$ & $\begin{array}{r}235 \\
83 \\
51 \\
39 \\
6 \\
11 \\
27 \\
61 \\
38 \\
108 \\
58 \\
37\end{array}$ & $\begin{array}{r}61 \% \\
21 \% \\
13 \% \\
10 \% \\
1 \% \\
3 \% \\
7 \% \\
16 \% \\
10 \% \\
28 \% \\
15 \% \\
10 \%\end{array}$ & $\begin{array}{r}13 \\
29 \\
9 \\
38 \\
36 \\
20\end{array}$ & $\begin{array}{r}52 \% \\
23 \% \\
10 \% \\
11 \% \\
\\
\\
9 \% \\
19 \% \\
6 \% \\
26 \% \\
25 \% \\
14 \%\end{array}$ & $\begin{array}{r}160 \\
49 \\
37 \\
23 \\
6 \\
11 \\
14 \\
32 \\
29 \\
70 \\
22 \\
17\end{array}$ & $\begin{array}{r}66 \% \\
20 \% \\
15 \% \\
10 \% \\
3 \% \\
5 \% \\
6 \% \\
13 \% \\
12 \% \\
29 \% \\
9 \% \\
7 \%\end{array}$ & $\begin{array}{c}0,006 \\
N S \\
N S \\
N S \\
N S \\
N S \\
N S \\
N S \\
N S \\
N S \\
<0,0001 \\
0,027\end{array}$ \\
\hline
\end{tabular}

NS: No significativo 
de la edad de éstos pacientes. La estancia media de ingreso fue significativamente superior en los pacientes $\geq 65$ años frente aquellos más jóvenes. La distribución por sexos fue de 283 varones $(73 \%)$ y 105 mujeres (23\%). La edad media fue de 66,3 años (rango 21-96, DS 14); más de la mitad (62,6\%) tenían 65 años o más. La edad media de los varones fue de 65,9 años y la de las mujeres 67,1 años. La sospecha de neoplasia al inicio del ingreso estuvo presente en 320 pacientes $(82,5 \%)$. La proporción de hombres y mujeres, así como la sospecha diagnóstica fue similar en los dos grupos de edad. El $61 \%$ de los pacientes presentó síndrome constitucional, que a su vez fue el principal motivo de ingreso (20\%). El síndrome constitucional fue más frecuente en los pacientes con edad $\geq$ 65 años, mostrando diferencias estadísticamente significativas respecto los más jóvenes $(\mathrm{p}=0,001)$. El dato más destacable de la exploración física fue la presencia de hepatomegalia (28\%). Las adenopatías y la focalidad neurológica fueron más frecuentes en los pacientes $<65$ años $(\mathrm{p}<0,05)$.

En la tabla II figuran las exploraciones complementarias realizadas más relevantes, exceptuando la analítica de rutina. La única exploración complementaria que se realizó a todos los pacientes fue la radiografía de tórax, que mostró hallazgos

\section{TABLA II}

\section{EXPLO RACIO NES CO M PLEM ENTARIAS REALIZADAS M ÁS RELEVANTES (EXCEPTUANDO ANALÍTICA)}

\begin{tabular}{lcccc}
\hline & \multicolumn{2}{c}{ Realizada } & \multicolumn{2}{c}{ Patológica } \\
& $n$ & $\%$ & $n$ & $\%$ \\
\hline Radiografía de tórax & 388 & 100 & 206 & 53 \\
TC torácica & 244 & 63 & 208 & 85 \\
TC abdominal & 210 & 54 & 154 & 73 \\
Ecografía abdominal & 174 & 45 & 124 & 71 \\
Broncoscopia & 89 & 23 & 63 & 71 \\
Fibrogastroscopia & 89 & 23 & 38 & 43 \\
Gammagrafía ósea & 86 & 22 & 51 & 59 \\
TC cerebral & 55 & 14 & 26 & 47 \\
Colonoscopia & 40 & 10 & 20 & 50 \\
Enema opaca & 40 & 10 & 17 & 42,5 \\
\hline
\end{tabular}

TC: tomografía computerizada patológicos en un $53 \%$ de los casos. La TC torácica fue la prueba que obtuvo más resultados orientativos sobre el diagnóstico (85\%), seguida de la TAC abdominal (73\%). El número y el tipo de exploraciones complementarias fue idéntico en los dos grupos de edad, con una media de 6 exploraciones por paciente (mínimo 3 - máximo 12, incluyendo analítica general y radiografía de tórax). Tampoco hubo diferencias en el método de obtener el diagnóstico anatomopatológico (citología vs biopsia). El diagnóstico histológico se realizó mediante biopsia en 142 pacientes $(36,6 \%)$, por citología en $201(51,8 \%)$ y por combinación de ambas en $45(11,6 \%)$.

En la tabla III se exponen los tipos de neoplasia. El cáncer de pulmón fue el más frecuente, que a su vez fue el principal en los varones $(48,4 \%)$. En las mujeres destacaron las neoplasias hematológicas $(25,7 \%)$. En 46 pacientes $(12 \%)$ el diagnóstico fue de cáncer de origen desconocido. La edad no se asoció con en el tipo de neoplasia excepto para el cáncer de pulmón, que fue más frecuente en los pacientes $<65$ años ( $44 \%$ frente $36 \%$ en los $\geq 65$ años, $\mathrm{p}=0,006$ ). El estadiaje de las neoplasias, exceptuando las hematológicas, queda reflejado en la tabla IV. El $83 \%$ de los enfermos se presentaron en estadíos avanzados de la enfermedad. La localización más frecuente de las metástasis fue la hepática $(20 \%)$, seguida de la ósea (13\%), pleural (8\%), cerebral $(6 \%)$ y pulmonar $(4 \%)$. La edad no demostró estar relacionada con el estadiaje.

En la tabla V se recoge el control clínico posterior al alta de los enfermos. Un $72 \%$ de los pacientes fueron derivados a especialistas en su patología neoplásica. Los pacientes $\geq 65$ años fueron controlados en un alto porcentaje por especialidades no oncológicas (medicina interna, medico de cabecera y PADES o Servicio de Asistencia Paliativa a Domicilio), mientras que los $<65$ años fueron remitidos con más frecuencia a Oncología y Hematología $(\mathrm{p}<0,05)$. En un $63 \%$ de casos se ofreció tratamiento con quimioterapia, radioterapia, cirugía o tratamiento hormonal. Un $37 \%$ fueron tributarios únicamente de medidas paliativas, destacando un alto porcentage en los pacientes $\geq 65$ años respecto los más jóvenes (50 y 37\% respectivamente, $\mathrm{p}=0,001$ )

Diecisiete pacientes (4\%) fallecieron durante el ingreso, de los cuales 14 eran $\geq 65$ años, aunque las diferencias no fueron significativas.

TABLA III

\begin{tabular}{|c|c|c|c|c|c|c|c|}
\hline \multicolumn{8}{|c|}{ TABLA III } \\
\hline \multicolumn{8}{|c|}{ TIPO DE NEO PLASIA } \\
\hline & \multicolumn{2}{|c|}{$\begin{array}{c}\text { Total } \\
n=388\end{array}$} & \multicolumn{2}{|c|}{$\begin{array}{c}\text { Hombres } \\
n=283\end{array}$} & \multicolumn{2}{|c|}{$\begin{array}{c}\text { M ujeres } \\
n=105\end{array}$} & $p$ \\
\hline Pulmón & 151 & $39 \%$ & 137 & $48 \%$ & 14 & $13 \%$ & $<0,001$ \\
\hline Hematológica* & 56 & $14 \%$ & 29 & $10 \%$ & 27 & $26 \%$ & $<0,001$ \\
\hline Colon y recto & 32 & $8 \%$ & 18 & $6 \%$ & 14 & $13 \%$ & 0,04 \\
\hline Hepatobiliar & 23 & $6 \%$ & 19 & $7 \%$ & 4 & $4 \%$ & NS \\
\hline Gástrica & 18 & $5 \%$ & 11 & $4 \%$ & 7 & $7 \%$ & NS \\
\hline Pancreas & 14 & $4 \%$ & 11 & $4 \%$ & 3 & $3 \%$ & NS \\
\hline Esófago & 6 & $1 \%$ & 6 & $2 \%$ & \multicolumn{2}{|c|}{0} & NS \\
\hline Ginecológica** & 14 & $4 \%$ & & & 14 & $13 \%$ & \\
\hline Próstata & 5 & $1 \%$ & 5 & $2 \%$ & & & \\
\hline O rigen desconocido & 46 & $12 \%$ & 30 & $11 \%$ & 16 & $15 \%$ & NS \\
\hline Otras & 23 & $6 \%$ & 17 & $6 \%$ & 6 & $6 \%$ & NS \\
\hline
\end{tabular}

*Linfoma no Hodgkin 33, M ieloma M últiple 10, Leucemia Linfática Crónica 4, Enfermedad de Hodgkin 4, Leucemia M ieloide Aguda 2, Leucemia M ieloide Crónica 1, Macroglobulinemia 1, Leucemia Linfática Aguda 1.**O vario 12, mama 1, cérvix 1 
TABLA IV

ESTADIAJE DE LAS NEO PLASIAS EXCEPTUANDO LAS HEM ATO LÓ GICAS $(n=332)$

\begin{tabular}{crr}
\hline & $n$ & $\%$ \\
\hline Localizado & 51 & 15 \\
I & 14 & 4 \\
II & 37 & 11 \\
Diseminado & 276 & 83 \\
III & 61 & 18 \\
IV & 215 & 65 \\
No estadiado & 5 & 2 \\
\hline
\end{tabular}

\section{DISCUSIÓN}

La patología neoplásica es una de las más prevalentes en los servicos de Medicina Interna (11). En nuestro servicio los pacientes diagnosticados de cáncer supusieron un $12 \%$ del total de los ingresos durante un período de 3 años, la sospecha inicial de neoplasia estuvo presente en un elevado número de casos $(82,5 \%)$ y la estancia media del ingreso fue de $10,8 \pm$ 7,8 días, siendo superior en los pacientes de mayor edad. En la serie de Urrutia A. y cols. (12) las enfermedades neoplásicas constituyeron un $7 \%$ de los ingresos durante un período de 2,5 años, la sospecha inicial de neoplasia estuvo presente en un $68,4 \%$ y la estancia media de ingreso fue de 13 días. De acuerdo con las estadísticas y registros sobre cáncer, encontramos una mayor incidencia de tumores en los hombres que en las mujeres (13-15).

El cáncer es una enfermedad relacionada con el envejecimiento y una de las principales causas de mortalidad y morbilidad en este segmento de la población $(16,17)$. De acuerdo con la literatura, en nuestra serie, más del $50 \%$ de las neoplasias tuvo lugar en los pacientes $\geq 65$ años (4-8). La edad media de nuestros enfermos con cáncer fue de 66,3 años, superior a la serie de Urrutia y cols. (12) (edad media 61,1 años).

La principal característica clínica y que a su vez fue el principal motivo de ingreso de nuestros pacientes fue la presencia de síndrome constitucional. Dicho síndrome, también conocido como síndrome anorexia-caquexia, constituye un importante factor de morbilidad y mortalidad en los enfermos con cáncer (18). Nuestros pacientes presentaron síndrome constitucional en un $61 \%$ de casos, resultado que concuerda con series previas, donde el síndrome constitucional se halla presente entre un 30 y un $87 \%$ de los pacientes afectos de cáncer $(19,20)$. Las características clínicas de los pacientes neoplásicos de mayor edad son diferentes de aquellos más jóvenes (21). En nuestra serie los $\geq 65$ años presentaron síndrome constitucional con una frecuencia significativamente superior que los más jóvenes. A pesar de ello no mostraron con más frecuencia síntomas asociados a la pérdida de peso, como los vómitos o la plenitud abdominal (22). Tampoco presentaron una mayor incidencia de neoplasias gástricas o pancreáticas, ni se presentaron en estadíos más avanzados de la enfermedad, hechos también relacionados a una mayor pérdida ponderal (23). Todo ello sugiere que existan otros factores asociados que contribuyan a una mayor frecuencia del síndrome constitucional, como son las enfermedades crónicas, los factores psicológicos y el estado socioeconómico (24).

La edad puede llegar a ser un factor de discriminación para estudiar y estadiar los pacientes con cáncer (25). En nuestra serie, el número y el tipo de exploraciones complementarias realizadas fue similar en los dos grupos de edad. La edad tampoco se relacionó con el estadiaje. Se realizaron una media de 6 exploraciones por paciente, incluyendo analítica general y radiografía de tórax, resultado que coincide con el estudio de Farag y cols. (26). De las exploraciones complementarias realizadas en nuestro estudio destacó el alto valor orientativo de pruebas tan sencillas como la radiografía de tórax (53\%) y la ecografía abdominal $(71 \%)$ y que podría ser explicado por la alta incidencia de neoplasias pulmonares y de metástasis hepáticas en nuestra serie. La tomografía computerizada ha demostrado su gran rentabilidad en el estudio de los pacientes con cáncer (27). En nuestro estudio, la TC abdominal y sobretodo la TC torácica demostraron una alta rentabilidad para llegar al diagnóstico.

La localización de los diferentes grupos de neoplasia no se vio influenciada por la edad de corte que establecimos en nuestros pacientes, pero sí, y como era de esperar, por el sexo. De acuerdo con las estadísticas sobre incidencia del cáncer en España (28), el cáncer más frecuente en los hombres fue el de pulmón (48\%). No fue así con las mujeres en quienes las neo-

TABLA V

CONTRO L CLÍNICO POSTERIOR AL ALTA

\begin{tabular}{|c|c|c|c|c|c|c|c|}
\hline \multirow[b]{2}{*}{ M edicina Interna } & \multicolumn{2}{|c|}{$\begin{array}{c}\text { Total } \\
n=388\end{array}$} & \multicolumn{2}{|c|}{$\begin{array}{c}\text { Edad }<65 \text { años } \\
n=145\end{array}$} & \multicolumn{2}{|c|}{$\begin{aligned} \text { Edad } & \geq 65 \text { años } \\
n & =243\end{aligned}$} & \multirow{2}{*}{$\begin{array}{c}p \\
<0,001\end{array}$} \\
\hline & 45 & $12 \%$ & 6 & $4 \%$ & 39 & $16 \%$ & \\
\hline O ncología & 175 & $45 \%$ & 82 & $57 \%$ & 93 & $38 \%$ & $<0,001$ \\
\hline Hematología & 48 & $12 \%$ & 26 & $18 \%$ & 22 & $9 \%$ & 0,02 \\
\hline M édico cabecera & 28 & $7 \%$ & 5 & $3 \%$ & 23 & $10 \%$ & 0,04 \\
\hline PADES & 18 & $5 \%$ & 1 & $1 \%$ & 17 & $7 \%$ & 0,009 \\
\hline Cirugía & 23 & $6 \%$ & 10 & $7 \%$ & 13 & $5 \%$ & NS \\
\hline O ncología + Cirugía & 24 & $6 \%$ & 7 & $5 \%$ & 17 & $7 \%$ & NS \\
\hline Ginecología & 6 & $2 \%$ & 3 & $2 \%$ & 3 & $1 \%$ & NS \\
\hline Urología & 4 & $1 \%$ & 2 & $1 \%$ & 2 & $1 \%$ & NS \\
\hline Exitus & 17 & $4 \%$ & 3 & $2 \%$ & 14 & $6 \%$ & NS \\
\hline
\end{tabular}

NS: no significativo 
plasias hematológicas ocuparon el primer lugar. La baja incidencia de cáncer de próstata en varones y de neoplasias ginecológicas en las mujeres de la presente serie podría ser explicada por el hecho que el estudio se ha realizado en un ámbito restringido a la medicina interna. Los tumores de origen desconocido supusieron un $12 \%$ del total, resultado que se asemeja a series previas, donde se estima una prevalencia entre el 3 y el $15 \%$ (29).

Un hallazgo muy relevante de nuestro estudio fue el alto porcentage de pacientes que se presentaron en estadíos avanzados de la enfermedad. A pesar que los pacientes mayores tienen más probabilidad de ser diagnosticados en fases diseminadas (30), en nuestra serie no hallamos diferencias en el estadiaje de las diversas neoplasias en los dos grupos de edad. La explicación a este hecho podría ser que a todos los pacientes se les ofreció un manejo diagnóstico y de estadiaje similar. Este hecho contrasta con la actitud terapéutica y el control posterior al alta que se les ofreció a nuestros pacientes de mayor edad, que en un 50\% de los casos fueron tratados de forma únicamente paliativa y en un alto porcentaje se desesti- mó un control posterior por especialidades oncológicas. Este hecho nos ha de hacer reflexionar que la edad por sí sola no ha de ser un factor descriminativo en los pacientes con cáncer y que tendría que ser tan sólo un parámetro más a tener en cuenta dentro de un enfoque individualizado y multidimensional $(21,31)$. De todo ello se deriva la necesidad de realizar estudios específicos sobre cáncer en gente mayor, de utilizar escalas de comorbididad, status funcional y social y de unificar criterios para crear unas guías comunes de manejo y de integrar los conocimientos de todos los clínicos que tratan con enfermos mayores (32).

Del presente trabajo queremos destacar la alta incidencia de enfermedad neoplásica en nuestro servicio de Medicina Interna, con un elevado porcentage de enfermos diagnosticados en fases avanzadas de la enfermedad, y que la edad no se relacionó con el manejo diagnóstico ni con el estadiaje, pero sí con la derivación al alta y en el tratamiento, de manera que los pacientes de 65 años o mayores fueron controlados con mayor frecuencia por especialialidades no oncológicas recibiendo en un alto porcentaje tratamiento unicamente paliativo.

\section{Bibliografía}

1. Boyle P. Global burden of cancer. Lancet 1997; 349 (Supl. 2): 23-26

2. Bailar J, Gornik H. Cancer undefeated. NEJM 1997; 336: 1569-1574.

3. Fraumeni JF, Devesa SS, Hoover R, Kinlen L. Epidemiology of cancer. In: De Vita V, Hellamn S. Rosemberg S, eds. Cancer Principles and Practice of Oncology. 4th de. Philadelphia, Pa: Lippincott Williams and Wilkins: 1994: 150-182.

4. Yancik R, Ries LAG. Cancer in the aged. An epidemiologic perspective on treatment issues. Cancer 1991; 68: 2502-2510.

5. Yancik R, Ries LAG. Aging and cancer in America. Demographic and epidemiologic perspectives. Hematol Oncol Clin North Am 2000; 14: 17-23

6. Trimble EL, Carter CL, Cain D, Freidlin B, Ungerleider RS, Friedman MA. Representation of older patients in cancer treatment trials. Cancer 1994; 74: 2208-14.

7. Hutchins LF, Unger JM, Crowley JJ, Coltman CA, Albain KS. Underrepresentation of patients 65 years of age or older in cancer-treatment trials. N Engl J Med 1999; 341: 2061-2067.

8. Kennedy BJ. Needed: Clinical Trials for older Patients. J Clin Oncol 1991; 9: 718-720.

9. Monfardini S, Aapro M, Ferrucci L, Zagonel V, Scalliet P, Fentiman I. Commision of the European Communities "Europe against cancer" Programme European School of Oncology Advisory Report Cancer Treatment in the Elderly. Eur J Cancer 1993; 29A: 2325-2330.

10. Repetto L, Venturino A, Vercelli M, Gianni W, Biancardi Vittorio, Casella C, et al. Performance Status and comorbidity in elerly cancer patients compared with young patients with neoplasia and elderly patients without neoplastic conditions. Cancer 1998; 82: 760-765.

11. García-Bragado F. Diagnóstico del cáncer: ¿qué, cómo, cuándo ? Med Clin (Barc) 1996; 106:695-696.

12. Urrutia A, Segura A, Monreal A, Cuxart A, Martí S, Rey-Joly C, Roca J. Análisis de la conducta diagnóstica de las neoplasias en un servicio de Medicina Interna. Med Clin (Barc) 1996; 106: 681-685.

13. Bernal M, Gómez E. Estudio de la mortalidad por cáncer. Año 1989. Neoplasia 1994; 11: 20-23.

14. Estapé A, Ribé A, Conesa A. Registro de tumores del Hospital Clínico y Provincial de Barcelona (1993). Neoplasia 1995; 12: 59-62

15. Moreno V, Sánchez V, Galcerán J, Borrás M, Borrás J, Bosch FX. Med Clin (Barc) 1998; 110: 86-93.

16. Yancik R. Cancer burden in the aged. An epidemiologic an demographic overview. Cancer 1997; 80: 1273-83.
17. Kennedy BJ. Aging and cancer. Geriatric oncology-Keynote address to the integrating geriatrics into oncology education. Cancer 1997; 80: 1270-1272.

18. Albretcht JT, Canada TW. Cachexia and anorexia in malignancy. Hematol Oncol Clin North Am 1996; 10: 791-800.

19. Dewis WD, Begg C, Lavin PT et al. Prognostic effect of weight loss prior to chemotrherapy in cancer patients. Am J Med 1980; 69: 491-497

20. Grosvenor M, Bulcavage L, Chlebowski RT. Symptoms potentially influencing weight loss in a cancer population. Correlations with primary site, nutritional status and chemotherapy administration. Cancer 1989; 63: 330-334.

21. Fírvida JL, Viñolas N, Muñoz M, Grau JJ, Daniels M, Estapé A et al. Age: a critical factor in cancer management. A prospective comparative study of 400 patients. Age and Ageing 1999; 28: 103-105.

22. Grosvenor M, Bulcavage L, Chlebowski RT. Symptoms potentially influencing weight loss in a cancer population. Correlations with primary site, nutritional status and chemotherapy administration. Cancer 1989; 63: 330-334.

23. Dewys W, Begg C, Lavin PT, et al. Prognostic effect of weight loss prior to chemotherapy in cancer patients. Am J Med 1980; 69: 491-497.

24. Fischer J, Johnson MA. Low body weight and weight loss in the aged. J Am Diet Assoc 1990; 90: 1697-1706.

25. Kennedy BJ. Aging and cancer. J Clin Oncol 1988; 6: 1903-1911.

26. Farag SS, Green MD, Morstyn G, Sheridan WP, Fox RM. Delay by Internists in obtaining diagnostic biopsies in patients with suspected cancer. Ann Int Med 1992; 116: 473-478.

27. Karsell PR, Sheeny PF, O,Conell M. Computed tomography in search of cancer of unknown origin. JAMA 1982; 248: 340-343.

28. Incidencia del cáncer en España. Distribución porcentual de los cánceres más frecuentes, 1990. JANO (Extra mayo) 1999; LVI (1303): 48.

29. Greco FA, Hainsworth JD. Cancer of unknown primary site, in De Vita VT Jr. Hellman S. Rosemberg SA (eds): Cancer: Principles and Practice of oncology (ed 4). Philadelphia, PA, Lippincott, 1993, pp 2072-92.

30. Extermann M, Aapro M. Assessment of the older cancer patient. Hematol Oncol Clin North Am 2000; 14: 63-77.

31. Balducci L, Extermann M. Cancer and aging. An evolving panorama. Hematol Oncol Clin North Am 2000; 14: 1-16.

32. Hazzard WR, Woolard N. Aging and cancer. Cancer (special issue) 1997; 80: 1267-1356. 